\title{
Managing anthracycline-induced cardiotoxicity: beginning with the end in mind
}
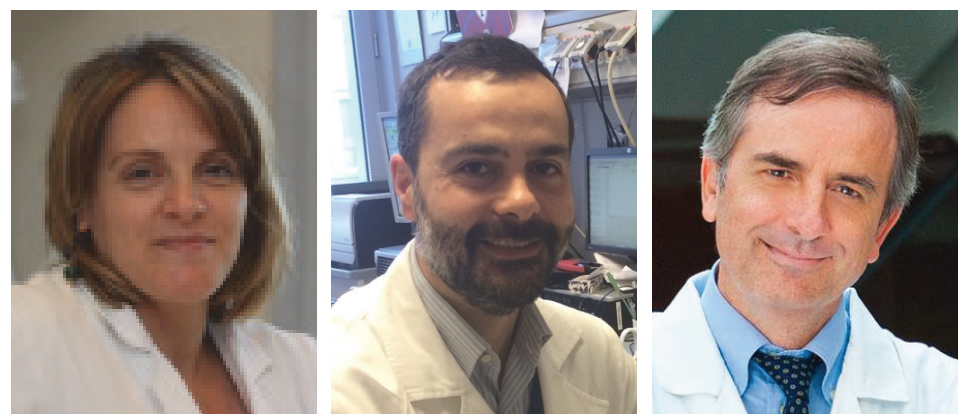

Emanuela Salvatorelli', Pierantonio Menna ${ }^{1,2}$ \& Giorgio Minotti ${ }^{* 1,2}$

Doxorubicin and other anthracyclines (epirubicin, daunorubicin, idarubicin) show superior antitumor activity in many solid or hematologic malignancies; however, anthracyclines have long been known to induce cardiotoxicity that may progress toward dilated cardiomyopathy and systolic heart failure. With each anthracycline analog the risk of heart failure correlates with the cumulative administered dose of that particular analog. In the case of doxorubicin, the risk of heart failure averages $5 \%$ at a cumulative dose of $400 \mathrm{mg} / \mathrm{m}^{2}$ but increases exponentially at doses higher than $500 \mathrm{mg} / \mathrm{m}^{2}[1]$.

Inasmuch as cardiotoxicity correlates with the cumulative anthracycline dose, one might easily conclude that limiting patient's exposure to, for example, $240-360 \mathrm{mg}$ of doxorubicin $/ \mathrm{m}^{2}$ should serve the most obvious and clinically doable means to avoid cardiac sequelae. Regrettably, however, this may not always be the case. Limiting doxorubicin cumulative dose to below $400 \mathrm{mg} / \mathrm{m}^{2}$ abates the risk of serious on-treatment cardiac events but not the risk of delayed cardiac events. Patients exposed to reportedly safe doses of anthracyclines may develop cardiomyopathy and heart failure anytime after cancer diagnosis and treatment, sometime decades after the last anthracycline administration [2]. Furthermore, some cancer survivors do not always present the canonical phenotype of dilated cardiomyopathy with systolic heart failure but may develop restrictive cardiomyopathy with diastolic heart failure or ischemic disease, particularly in cases when chemotherapy was followed by supradiaphragmatic radiotherapy [3].

Many other aspects of anthracyclineinduced cardiotoxicity remain undecipherable. Additive or synergic interactions of anthracyclines with other drugs have been documented or surmised but substantial individual variation in vulnerability and clinical outcome is also evident [2]. Molecular foundations of early or delayed cardiac events are only in part understood, presumably because cardiotoxicity is a multifactorial process that cannot be recapitulated under the umbrella of just a single mechanistic hypothesis [4]. And finally, full blown anthracycline-induced heart failure is plagued by higher rates of mortality as

\section{KEYWORDS \\ - anthracyclines \\ - cardiotoxicity \\ - comorbidities \\ - dexrazoxane - doxorubicin \\ - heart failure - prevention \\ - surveillance • survivors}

“...anthracyclines have long been known to induce cardiotoxicity that may progress toward dilated cardiomyopathy and systolic heart failure."

'Unit of Drug Sciences, Department of Medicine, University Campus Bio-Medico, Via Alvaro del Portillo 21, 00128 Rome, Italy

2Unit of Clinical Pharmacology, Campus Bio-Medico University Hospital, Via Alvaro del Portillo 200, 00128 Rome, Italy *Author for correspondence: Tel.: +39 06 225419109; Fax: +39 06 22541456; g.minotti@unicampus.it

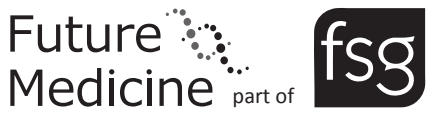




\section{“Optimal management of anthracycline-induced cardiotoxicity ... calls primarily for risk} assessment and mitigation and hence, for primary and secondary prevention." compared with heart failure from other causes [5]. This having been said, one cannot escape the conclusion that there is no completely 'safe' dose of anthracyclines in either pediatric or adult settings. Some patients may be genetically predisposed to a higher risk of cardiotoxicity, but there is a lack of studies that prospectively characterized the predictive value of genetic tests [6] Optimal management of anthracycline-induced cardiotoxicity therefore calls primarily for risk assessment and mitigation and hence, for primary and secondary prevention.

Primary prevention begins with a rigorous patient's assessment. Comorbidities (hypertension, arrhythmias, diabetes, hyperlipidemia, reduced physical activity and overweight) increase patient's vulnerability by reportedly safe cumulative doses of anthracyclines [2]. In approaching this constellation of factors, clinicians should assess risk versus benefit and avoid decisions that invalidate the life-saving effects of anthracyclines. Switching from anthracyclines to other drugs, or reducing cumulative anthracycline doses to below a threshold that proved to be active and safe in low risk patients, might cause tumor undertreatment and poor oncologic outcome $[4,6]$. Pharmacologic and behavioral correction of comorbidities and risk factors are cornerstone of optimal management of at-risk patients and may allow for delivering cumulative anthracycline doses that are needed to cure cancer in these patients.

Clinicians should also consider adopting strategies that reduce cardiac exposure to anthracyclines or interfere with mechanisms of anthracycline toxicity in the cardiac tissue. Blunting the plasma peak concentration of anthracyclines reduces cardiac exposure to anthracyclines [7]. One may achieve this goal by replacing bolus administration with slow infusions or by replacing conventional anthracyclines with liposome-encapsulated anthracyclines [6]. On the other hand, one or more mechanisms of anthracycline cardiotoxicity can be interrupted by coadministering dexrazoxane, the only drug that granted approval from US FDA for use as cardiac protectant in patients exposed to anthracyclines $[4,6,7]$.

But not all that glitters is gold. Each of the aforesaid measures of primary prevention suffers from limitations or conceptual biases that need to be scrutinized and managed in the interest of patient's optimal treatment. Slow infusions are cardioprotective in many pediatric and adult oncologic settings but not in some defined diseases, for example, pediatric acute lymphoblastic leukemia [8]; moreover, switching from bolus administration to prolonged infusions introduces both the added costs of longer hospital stays and the increased risk of infections and mucositis [9]. Liposomal anthracyclines are approved for use in only limited settings, which makes them too expensive to use in some countries [6]. As to dexrazoxane, we still do not know how precisely it protects the heart; moreover, its popularity was limited by concerns that it interfered with anthracycline activity [10] or caused an increased risk of secondary malignancies [11]. Either concern proved to be unjustified or overemphasized, and yet both the US FDA and the EMA introduced a number of caveats that limited dexrazoxane indications in adult or pediatric patients [12]. On balance, we believe that risk versus benefit assessment and cost-effectiveness analysis outweighs concerns or biases and support a wider usage of liposomal anthracyclines or dexrazoxane.

Managing anthracycline cardiotoxicity is also a matter of secondary prevention. Patients should be monitored during therapy and 'early signals' of cardiotoxicity should be promptly managed. With that said, how can early cardiotoxicity be defined? Clinical symptoms of heart failure represent cardiotoxicity by definition but asymptomatic decreases of left ventricular ejection fraction (LVEF) occur more often and do not always herald authentic anthracycline toxicity. In some patients, LVEF returned to baseline if chemotherapy was stopped but in other patients LVEF returned to baseline also when chemotherapy was continued [13]. Many factors (hydration status, anemia, infections, neurohumoral changes) can transiently influence LVEF independent of any ongoing damage from anthracyclines [6]. If such transient decreases of LVEF were used to reduce anthracycline dose intensity or to replace anthracyclines with other drugs, patients will be exposed to the risk of undertreatment and low oncologic efficacy. On the other hand, limited studies suggest that patients with normal LVEF may show asymptomatic diastolic dysfunction or elevations of circulating troponin that are more likely to herald anthracycline-related cardiotoxicity and possibly identify patients at a higher risk of subsequent decreases of LVEF or heart failure [2].

In awaiting for larger studies that confirmed the prognostic value of early diastolic dysfunction or troponin elevations, one might ask about actions to take in asymptomatic patients 
presenting with one or both these laboratory findings. Early commencement of enalapril, as guided by troponin elevations, was shown to protect patients against delayed decreases of LVEF [14]. Other studies are probing the value of an early medication of chemotherapy-induced diastolic dysfunction [15], while many more studies are probing the benefit of starting $\beta$-blockers and/or angiotensin converting enzyme inhibitors even before chemotherapy was started [16]. All such approaches rest with the assumption that subclinical cardiotoxicity develops after few doses of anthracyclines and calls for the earliest possible commencement of cardiovascular drugs. There is no formal guideline or recommendation to support this strategy, particularly in patients without risk factors. We nonetheless believe that common sense does support early cardiovascular protection. Prospective multicenter studies with a 'real life' design that include both low- and high-risk patients are much needed to bring common sense to the level of clinical evidence and recommendations.

Post-chemotherapy surveillance is the last available chance to detect subclinical or mild asymptomatic cardiotoxicity before it progressed toward potentially fatal heart failure. Each patient should be given a well-defined program of surveillance by dedicated experts. Comorbidities come again into play as cancer survivors tend to develop new comorbidities or may experience a worsening of pre-existing comorbidities. There is a persuasive evidence that progression of cardiotoxicity from subclinical damage to mild symptomatic cardiotoxicity, and eventually to heart failure, is driven by a conspiracy of anthracycline-dependent toxicity with metabolic or hemodynamic stress from comorbidities [17]. This complex scenario may require that cardiologists and oncologists collaborate with other key figures (endocrinologists, pharmacologists, nutritionists) and design surveillance or treatment modalities that best suit the pathophysiology of a patient or another [18].

Anthracyclines have been around for decades now and some believe that they are too old to be good and compare with forthcoming avalanches of newer drugs. Even certain combinations of conventional chemotherapeutics were reported to show similar efficacy but lower cardiotoxicity than doxorubicin [19]. This having been recognized, choosing between anthracyclines and nonanthracycline drugs remains a matter of debate [20]. While the jury is still out, the only means to exploit anthracyclines at their best therapeutic performance is to begin treatment with the end in mind, for example, to cure patients while also devising primary and secondary measures that prevented or delayed cardiac events for as long as possible.

Financial \& competing interests disclosure

G Minotti reports receiving lecture fees from Teva and Cell Therapeutics BioPharma, and grant support through his institution from Menarini International Operations Luxembourg. The authors have no other relevant affiliations or financial involvement with any organization or entity with a financial interest in or financial conflict with the subject matter or materials discussed in the manuscript apart from those disclosed.

No writing assistance was utilized in the production of this manuscript.

\section{References}

Papers of special note have been highlighted as: - of interest

1 Swain SM, Whaley FS, Ewer MS. Congestive heart failure in patients treated with doxorubicin: a retrospective analysis of three trials. Cancer 97(11), 2869-2879 (2003).

2 Minotti G, Salvatorelli E, Menna P. Pharmacological foundations of CardioOncology. J. Pharmacol. Exp. Ther. 334(1), 2-8 (2010).

3 Swerdlow AJ, Higgins CD, Smith P et al. Myocardial infarction mortality risk after treatment for Hodgkin disease: A collaborative British Cohort Study. J. Natl Cancer. Inst. 99(3), 206-214 (2007).
4 Yeh ET, Salvatorelli E, Menna P, Minotti G. What is cardiotoxicity? Progr. Pediatr. Cardiol. 36(1-2), 3-6 (2014).

- Experts in preclinical and clinical research show that a precise definition of cardiotoxicity is still lacking, which may complicate decisions about using or avoiding anthracycline-based treatment protocols.

5 Von Hoff DD, Layard MW, Basa P et al. Risk factors for doxorubicin-induced congestive heart failure. Ann. Intern. Med. 91(5), 710-717 (1979).

6 Salvatorelli E, Menna P, Cantalupo E et al. The concomitant management of cancer therapy and cardiac therapy. Biochim. Biophys. Acta doi: 10.1016/j.bbamem.2015.01.003 (2015) (Epub ahead of print).
- Contemporary review that summarizes classical or more recent mechanistic hypotheses of anthracycline-induced cardiotoxicity and suggests what can be done in the settings of primary or secondary prevention and surveillance.

7 Minotti G, Menna P, Salvatorelli E, Cairo G, Gianni L. Anthracyclines: molecular advances and pharmacologic developments in antitumor activity and cardiotoxicity. Pharmacol. Rev. 56(2), 185-229 (2004).

8 Lipshultz SE, Miller TL, Lipsitz SR et al. Continuous versus bolus infusion of doxorubicin in children with ALL: long-term cardiac outcomes. Pediatrics 130(6), 1003-1011 (2012). 
9 Menna P, Gonzalez Paz O, Chello M et al. Anthracycline cardiotoxicity. Expert. Opin. Drug. Saf. 11(Suppl. 1), S21-S36 (2012).

10 Swain SM, Whaley FS, Gerber MC et al. Cardioprotection with dexrazoxane for doxorubicin-containing therapy in advanced breast cancer. J. Clin. Oncol. 15(4), 1318-1332 (1997).

11 Tebbi CK, London WB, Friedman D et al. Dexrazoxane-associated risk for acute myeloid leukemia/myelodysplastic syndrome and other secondary malignancies in pediatric Hodgkin's disease. J. Clin. Oncol. 25(5), 493-500 (2007).

12 Lipshultz SE, Franco VI, Sallan SE et al. Dexrazoxane for reducing anthracyclinerelated cardiotoxicity in children with cancer: an update of the evidence. Progr. Pediatr. Cardiol. 36(1-2), 39-49 (2014).

- Highly recommended review that describes clinical evidence of cardioprotection by dexrazoxane, relevant regulatory issues, recommendations for avoiding late cardiovascular events in childhood cancer survivors.
13 Wouters KA, Kremer LC, Miller TL et al. Protecting against anthracycline-induced myocardial damage: a review of the most promising strategies. Br. J. Haematol. 131(5), 561-578 (2005).

14 Cardinale D, Colombo A, Sandri MT et al. Prevention of high-dose chemotherapyinduced cardiotoxicity in high-risk patients by angiotensin-converting enzyme inhibition. Circulation 114(23), 2474-2481 (2006).

- Seminal work on troponin elevations to identify patients at risk for systolic dysfunction and to commence cardiovascular protection with angiotensin converting enzyme inhibitors.

15 Minotti G. Pharmacology at work for cardio-oncology: ranolazine to treat early cardiotoxicity induced by antitumor drugs. J. Pharmacol. Exp. Ther. 346(3), 343-349 (2013).

16 Vejpongsa P, Yeh ETH. Prevention of anthracycline-induced cardiotoxicity J. Am. Coll. Cardiol. 64(9), 938-945 (2014).
17 Menna P, Salvatorelli E, Minotti G. Cardiotoxicity of antitumor drugs. Chem. Res. Toxicol. 15(9), 1179-1189 (2008).

18 Nathan PC, Ford JS, Henderson TO et al. Health behaviors, medical care, and interventions to promote healthy living in the Childhood Cancer Survivor Study cohort. J. Clin. Oncol. 27(14), 2363-2373 (2009).

19 Jones SE, Holmes FA, O'Shaughnessy JA et al. Docetaxel with cyclophosphamide is associated with an overall survival benefit compared with doxorubicin and cyclophosphamide: 7 year follow-up of US Oncology Research Trial 9735. J. Clin. Oncol. 27(8), 1177-1183 (2009).

20 Gianni L, Valagussa P. Anthracyclines and early breast cancer: the end of an era? J. Clin. Oncol. 27(8), 1155-1157 (2009).

- Provocative editorial on the past and future of anthracyclines in the settings of adjuvant therapy of early breast cancer. A brief but insightful analysis of clinical evidence that supports or disproves the higher efficacy of anthracyclines in comparison with less cardiotoxic chemotherapeutics. 